\title{
A novel de novo variant of $L A M A 2$ contributes to merosin deficient congenital muscular dystrophy type 1A: Case report
}

\author{
KIEN TRUNG TRAN ${ }^{1}$, VINH SY LE ${ }^{1,2}$, CHINH DUY VU ${ }^{3}$ and LIEM THANH NGUYEN ${ }^{1}$ \\ ${ }^{1}$ Vinmec Research Institute of Stem Cell and Gene Technology, \\ Vinmec International Hospital; ${ }^{2}$ University of Engineering and Technology, Vietnam National University; \\ ${ }^{3}$ Vinmec Times City International Hospital, Vinmec International Hospital, Hanoi 100000, Vietnam
}

Received June 26, 2019; Accepted October 25, 2019

DOI: $10.3892 /$ br.2019.1260

\begin{abstract}
Merosin deficient congenital muscular dystrophy type $1 \mathrm{~A}$ (MDC1A) is caused by defects in the LAMA2 gene. Patients with MDC1A exhibit severe symptoms, including congenital hypotonia, delayed motor development and contractures. The present case report describes a Vietnamese male child with clinical manifestations of delayed motor development, limb-girdle muscular dystrophy, severe scoliosis and white matter abnormality in the brain. Whole exome sequencing (WES) was performed with subsequent validation using Sanger sequencing, and a de novo missense variant (NM_000426.3:c.1964T $>$ C, p.Leu655Pro) and a splice site variant (NG_008678.1:c.3556-13T $>A$ ) in the LAMA2 gene of the proband was detected. The missense variant located in exon 14 and has not been reported previously, to the best of our knowledge; whereas the splice site variant has been previously reported to cause premature termination of transcription in patients with MDC1A. In silico tools predicted that the missense variant was damaging. Phenotype-genotype analysis suggested that this proband was associated with classical early onset MDC1A. The co-existence of a de novo and a heterozygous variant in the LAMA2 gene suggested that the de novo variant contributed to the autosomal recessive manner of the disease. Careful consideration of this event by clinical confirmation of parental carrier status may help to accurately determine the risk of occurrence of this disease in future offspring. Additionally, WES is recommended as a powerful tool to assist in identifying potentially causative variants for heterogeneous diseases such as MDC1A.
\end{abstract}

Correspondence to: Dr Kien Trung Tran, Vinmec Research Institute of Stem Cell and Gene Technology, Vinmec International Hospital, 458 Minh Khai, Hai Ba Trung, Hanoi 100000, Vietnam E-mail: v.kientt10@vinmec.com

Key words: LAMA2 gene, de novo, whole exome sequencing, merosin deficient congenital muscular dystrophy type $1 \mathrm{~A}$

\section{Introduction}

Merosin deficient congenital muscular dystrophy type 1A (MDC1A) or laminin- $\alpha 2$ related muscular dystrophy (OMIM entry, \#607855) is one of the most common forms of congenital muscular dystrophy (CMD), and accounts for $30 \%$ of all cases of CMD in European countries (1). CMD may appear during early onset or later in a patient's life, and is characterized by proximal weakness, contractures, delayed motor development, white matter abnormalities and spiral scoliosis $(2,3)$. CMD is a hereditary disorder caused by recessive mutations in the LAMA2 gene, which is located on chromosome $6 \mathrm{q} 22-2$, spans over $260 \mathrm{~kb}$ and is comprised of 65 exons. LAMA2 encodes the laminin- $\alpha 2$ chain which attaches with laminin- $\beta 1$ and laminin- $\gamma 1$ chain to form the heterotrimeric protein laminin 211. Laminin 211 is a primary component of the skeletal muscle basement membrane and the extracellular matrix $(1,4,5)$. The protein interacts with other matrix macromolecules and contributes to cell differentiation, cell movement and tissue phenotypes (4). The majority of reported genetic variations of MDC1A are homozygous or compound heterozygous variants $(6,7)$. De novo variations in contrast are not common events and only a few have been reported in patients with MDC1A (8-10).

It is estimated that the prevalence of $\mathrm{MDC} 1 \mathrm{~A}$ is $1-9 / 1,000,000$ individuals, and accounts for 1-6\% of all CMD cases $(1,2)$. MDC1A is more common in Caucasians and rarer in the Asian population (2). However, the prevalence of the disease in the Asian population may be higher than expected due to a lack of widespread availability of appropriate diagnostic testing (11). In the present study, the case of a Vietnamese male child exhibiting clinical signs of muscular dystrophy is reported. The child was previously undiagnosed due to his rare clinical presentation and a lack of appropriate testing. Whole exome sequencing (WES) was performed on the patient and his parents. WES showed that the proband harbored two variants in the LAMA2 gene. Genotype-phenotype analysis suggested that the patient had classical early onset MDC1A. Additionally, the de novo variant was suggested to serve a contributive role in the development of the disease. To the best of our knowledge, the present study is the first to report a case of MDC1A in a Vietnamese patient. 


\section{Patients and methods}

Sample collection. Prior to collection of samples, written informed consent form was obtained from the parents for research collection and use of biological samples (blood and muscle) and clinical information including the results of the magnetic resonance imaging (MRI) scan, muscle biopsies and genetic testing. The present study was approved by the Institutional Ethical Review Board of Vinmec International General Hospital (Hanoi, Vietnam). From both the patient and his parents, $\sim 2 \mathrm{ml}$ of peripheral blood was collected. Blood samples were stored in EDTA containing tubes at $-80^{\circ} \mathrm{C}$. The deltoid was chosen as the muscle to obtain a biopsy from, and a standard procedure was followed (12).

Muscle pathological analysis. To examine the morphology of the muscle, muscle tissue sections were obtained, and were fixed and stained using hematoxylin and eosin staining, as described previously (13). Needle electromyography (EMG) was performed at the quadriceps and gastrocnemius muscle using a Nicolet EDX system (Natus Medical, Inc.).

WES. Genomic DNA was extracted from peripheral blood lymphocytes using a DNA Mini Blood Isolation kit according to the manufacturer's protocol (Qiagen $\mathrm{GmbH}$ ). A total of $50 \mathrm{ng}$ genomic DNA was used for library construction using a Nextera Rapid Capture kit (Illumina, Inc.) according to the manufacturer's protocol. Paired-end sequencing with a read length of $75 \times 2$ bp was performed using a HiSeq 4000 (Illumina, Inc.).

Variant identification and analysis. Burrows Wheeler Aligner (14) was used to map short reads to the human reference genome (GRCh37). Platypus program version 0.8.1 (15) and Genome Analysis Toolkit version $3.6(16,17)$ were both used for variant calling. Variants with a minor allele frequency $>1 \%$, as reported in the 1000 Genomes Project database (18), were removed. SnpEff program version $4.3 \mathrm{~g}$ (19) and the Human Genome Variant Database (20) were used for variant annotation. PolyPhen-2 (21) and PROVEAN version 1.13 (22) were both used to predict the impact of missense variant. PolyPhen-2 presents the damaging score as a value between 0 and 1 , where a score closer to 1 indicates a high probability of the substitution being damaging. For PROVEAN, if the score is $\leq-2.5$ (which is used as a threshold), this indicates the variant is damaging. Protein structure analysis was performed using HOPE (23).

Sanger validation. The candidate variants from WES were confirmed using Sanger sequencing on an ABI 3500 DX system with BigDye Terminator version 3.1 (Thermo Fisher Scientific, Inc.). The primer sequences used for validation were: NM_000426.3:c.1964T>C forward, 5'-TGAGGGTGG AGGATACAAATATAG-3' and reverse, 5'-AGGGCTCCGTT CTTATCTGC-3'; and NG_008678.1:c.3556-13T>A forward, 5'-AAGATTTACGTCCTGCCATGC-3' and reverse, 5'-CCA TGTGGGCAACAATCTCTG-3'.

\section{Results}

Case presentation. The proband was a male, born by caesarean section at 36 weeks of gestation to healthy and
Table I. Clinical features of the patient.

\begin{tabular}{ll}
\hline Age of onset & At birth \\
Age when diagnosed & 14 years old \\
Sex & Male \\
Ethnic group & Kinh Vietnamese \\
Height & $146 \mathrm{~cm}$ \\
Weight & $44.2 \mathrm{~kg}$ \\
Brain MRI & WMH on T2-weighted image \\
Mental retardation & No \\
Independent ambulation & No \\
Facial dysmorphy & No \\
Motor milestone & Sat supported \\
Contractures & Yes \\
Scoliosis & Yes \\
EMG myopathic changes & Yes \\
Creatine kinase & 126 U/1 \\
\hline
\end{tabular}

${ }^{a}$ Reference for merosin deficient congenital muscular dystrophy type $1 \mathrm{~A}<170 \mathrm{U} / 1$. WMH, abnormal brain white matter hyperintensity on T2W; EMG, electromyography; MRI, magnetic resonance imaging.
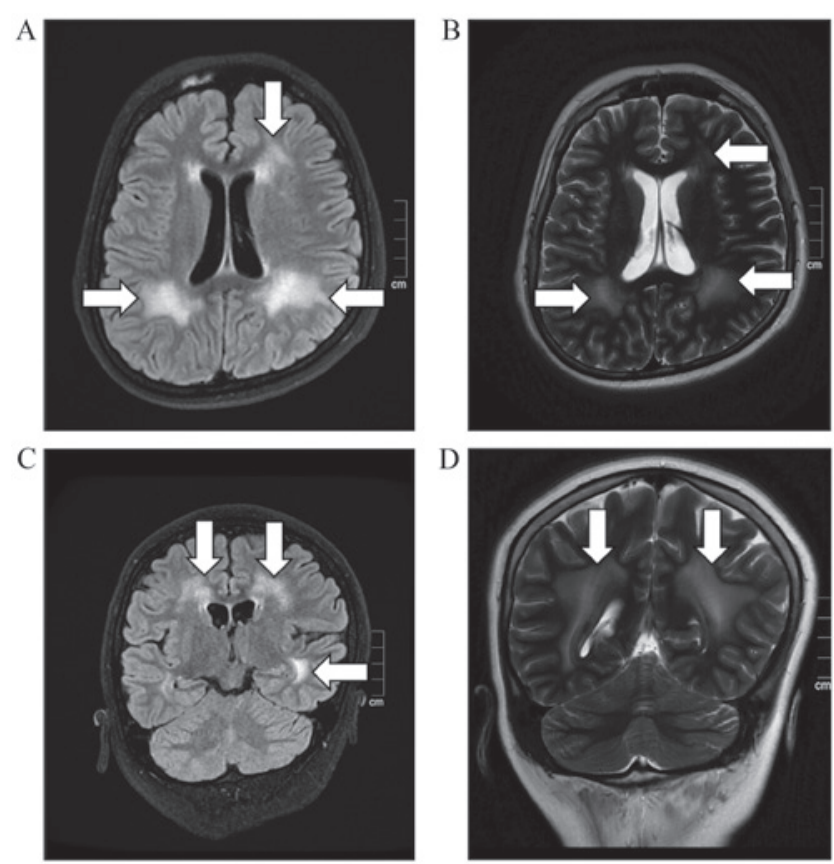

Figure 1. Magnetic resonance imaging results of the patient at the age of 14 years old. (A) Axial FLAIR. (B) Axial T2-weighted image. (C) Coronal FLAIR image. (D) Coronal T2-weighted image. Empty arrows present the brain lesions. FLAIR, fluid attenuated inversion recovery image.

non-consanguineous Vietnamese parents with no family history of any inherited disease. His weight was $3.4 \mathrm{~kg}$ and was considered as of no clinical concern at birth. The boy was reported to show a delay in motor milestone acquisition during his life. When visiting our hospital, the proband was 14 years old and he presented with gross motor developmental delay, severe spiral scoliosis and limb-girdle muscular dystrophy. He showed normal intelligence for his age, without any sign of ophthalmoplegia or elongated 

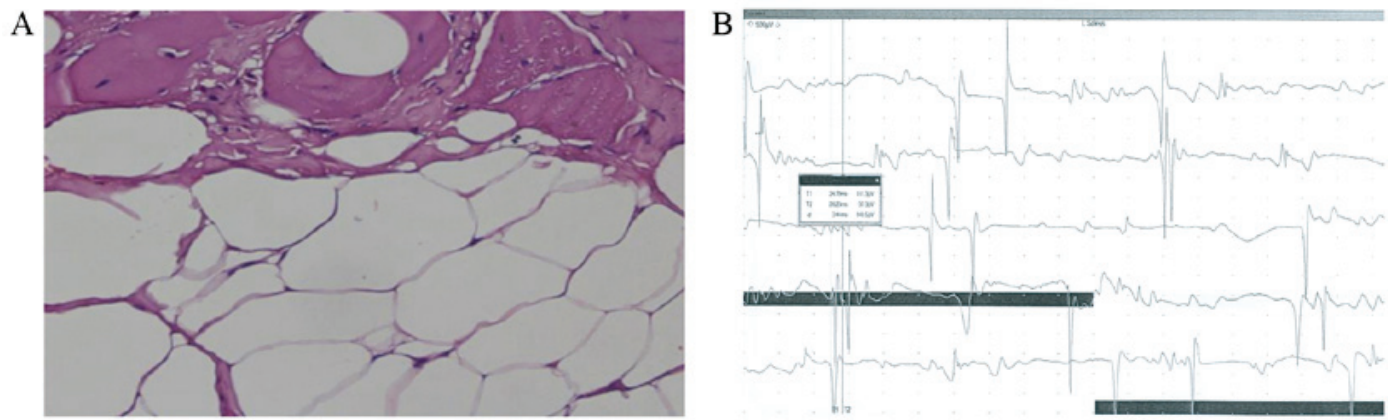

Figure 2. Muscle pathological analysis. (A) Hematoxylin and eosin staining of a muscle biopsy from the deltoid. Magnification, x400. (B) Morphology and recruitment pattern of motor unit action potential.

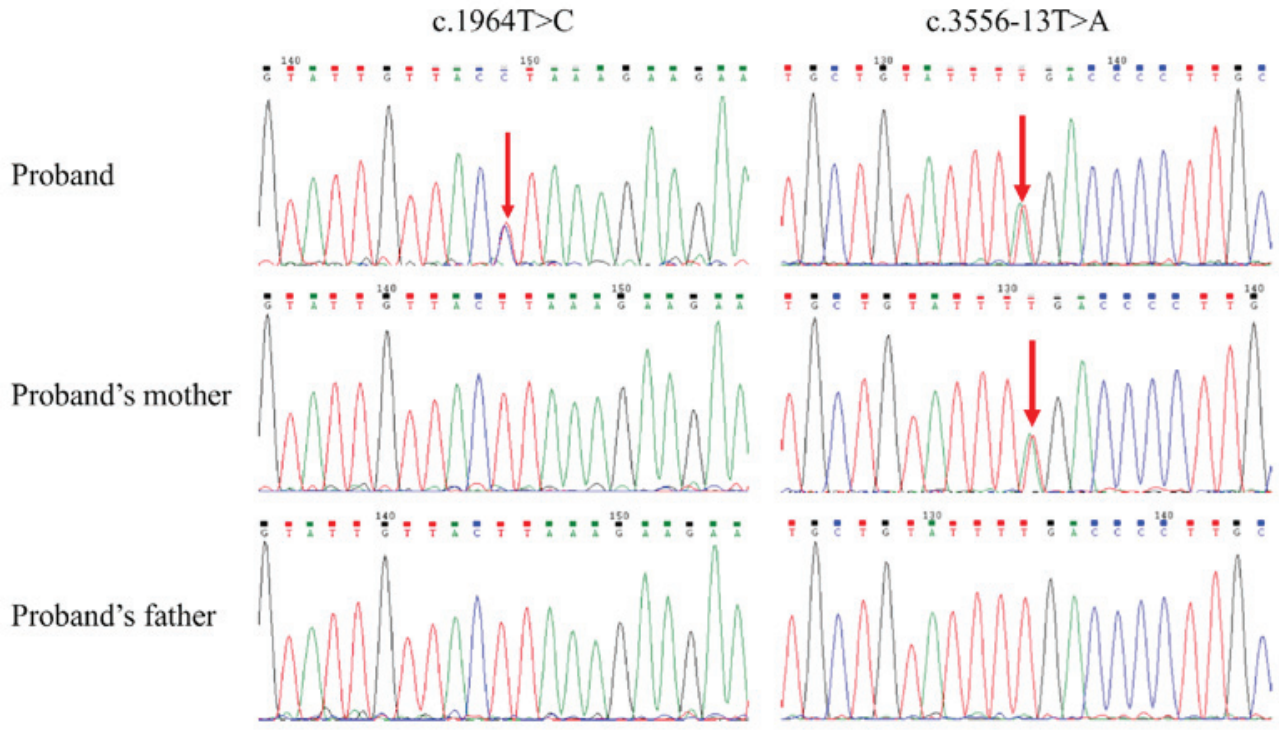

Figure 3. LAMA2 gene sequence of the proband and the parents. The missense variant, NM_000426.3:c.1964T>C, was only observed in the proband. The intronic variant, NG_008678.1:c.3556-13T>A, was observed in the proband and the mother. Red arrows show the position and heterozygosity of the variation.

face and could speak normally. The creatine kinase (CK) levels were normal (126 U/l; Table I). The boy was not able to sit or ambulate unsupported. A brain MRI scan revealed an increased signal at the frontal and the bilateral occipital lobe. Diffuse brain white matter hypointensity was also observed (Fig. 1). Deltoid muscle biopsy indicated fibrous-adipose replacement (Fig. 2A). In addition, needle EMG showed the motor units exhibited clear characteristics of myopathy (Fig. 2B) (24).

To evaluate gross motor functions, several tools, including the Gross Motor Function Classification System (GMFCS) $(25,26)$, Gross Motor Function Measure 88 scale (GMFM-88) for children with cerebral palsy aged 12-18 years old (27), and the modified Ashworth Scale were used to measure muscle spasticity (28). The proband was scored at level V based on the GMFSC scale, meaning that he had severe limitations and entirely relied on a wheelchair for movement. GMFM- 88 evaluation showed that the patient achieved 38 points in domain 'Lying and Rolling', 31 points in domain 'Sitting', one point in domain 'Crawling and Kneeling', and zero points in domains 'Standing', 'Walking', 'Running' and 'Jumping'. Modified Ashworth evaluations indicated that the scores of the upper and lower limbs ranged from 3-4 points, indicating that there was a considerable increase in muscle tone, and rigidity in flexion or extension.

Genetic studies. A total of 110, 86 and 101 million paired-end reads were obtained from the proband, and the father and the mothers genomes, respectively, where $95 \%$ of the reads had a Phred-score $\geq 30$ (95\% of the reads were called with a correct probability $\geq 99.9 \%$ ) (29). Average coverage at the targeted regions were $81 \mathrm{X}, 66 \mathrm{X}$ and $80 \mathrm{X}$ for the proband, father and mother, respectively. Two variants were detected in the LAMA2 gene (Reference sequence: NM_000426.3) of the proband and these variants were not previously described in the Vietnamese genetic variation database (genomes. $\mathrm{vn} /)(30)$ indicating their rare frequency in the population. Of these, the missense variant, NM_000426.3:c.1964T $>$ C, p.Leu655Pro, located in exon 14, resulted in a substitution of leucine to proline at the 655th amino acid residue. It appeared as a de novo variant as it was not observed in the parents. This variant has been not listed in the Leiden Open Variation Database (databases.lovd.nl/shared/genes/LAMA2) or elsewhere suggesting its novelty. In addition, WES results indicated that the proband and his mother carried a splice site variant (NG_008678.1:c.3556-13T>A) which was present 
at the intron-exon boundary of exon 25 of the LAMA2 gene. Sanger sequencing confirmed the findings from WES where the missense variant (NM_000426.3:c.1964T>C) was only detected in the proband; whereas the splice site variant (NG_008678.1:c.3556-13T>A) was found in the proband and his mother, but not in the father (Fig. 3). The splice site variant has been previously observed in two Chinese patients with MDC1A and is reported to cause mRNA splicing (6).

In silico predictions. PolyPhen-2 predicted that the missense variant (NM_000426.3:c.1964T>C) was damaging with a score of 1 . PROVEAN predicted that this variant was 'deleterious' with a score of -6.992 . Additionally, protein structure analysis using HOPE suggested that the missense mutant, the size of which was smaller compared with the wildtype, may result in a loss of amino acid interactions. Thus, a substitution of leucine to proline may disturb a functional domain of the laminin protein and abolish the proteins function.

\section{Discussion}

In the present study, two variations in the LAMA2 gene of a male proband who exhibited clinical manifestations of CMD were identified. The missense variant (NM_000426.3:c.1964T>C) is located in the $\mathrm{N}$-terminal domain of laminin- $\alpha 2$. A previous study found that half of the variants detected in a cohort of 43 patients with MDC1A were located in the N-terminal domain (6). Therefore, this may suggest that this domain is either more vulnerable than the other domains to mutations, is more sensitive to mutations or that it exhibits a crucial function on the part of the protein. WES analysis of the proband and his parents alongside database analyses indicated that NM_000426.3:c.1964T>C was de novo and a novel variant. A splice site variant (NG_008678.1:c.3556-13T>A) in the proband and his mother was also detected. This variant and its mRNA splicing effect (premature termination of transcription) have been previously described in two Chinese patients with MDC1A (6).

Together, these two variants likely caused a deficit in laminin- $\alpha 2$ function, chain which is a primary component of a trimeric basement membrane glycoprotein $(1,4,5)$. Mutations in the LAMA2 gene result in defective function of the laminin protein with consequential poor muscle fiber adhesion and degeneration (31). Phenotype-genotype analysis indicated that this proband was associated with classical early onset LAMA2-associated muscular dystrophy. Therefore, these two variants were submitted to the Global Variome Shared LOVD database (databases.lovd.nl/shared/individuals/00208524).

The missense variant was searched against in multiple databases and was not found to have been previously reported. Therefore, the present case report may be the first report underlining genetic variations in a Vietnamese individual with MDC1A. In addition to the rare nature of MDC1A, a lack of common symptoms in the child, such as increased levels of CK and ophthalmoplegia, and the genetic heterogeneity of this disease made diagnosis difficult. CK levels are often elevated in patients with MDC1A during the early years of a patient's life and tend to decrease with age (32). The proband in the present report exhibited normal levels of CK at the age of 14. Several studies have reported normal $\mathrm{CK}$ levels in patients with $\operatorname{MDC1A}(6,11,33)$. Therefore, age should be taken into account when interpreting the
CK level in each patient with CMD (34). In the present study, the patient was suspected to suffer from X-linked adrenoleukodystrophy owing to his abnormal brain MRI scan. Genetic testing of the $A B C D 1$ gene, defects of which cause X-linked adrenoleukodystrophy were performed. However, there was no evidence of mutations found and the patient remained undiagnosed prior to WES testing (data not shown).

MDC1A exhibits a wide spectrum of genetic and clinical heterogeneity. Immunohistochemistry (IHC) is a first-tier test used to determine CMD-associated diseases. Commercially available antibodies, such as clone 5H2, clone Mer3/22B2 and clone 4H8-2 are available for performing laminin protein assays (7). However, as MDC1A is extremely rare in Asian populations, including the Vietnamese, IHC using these specific antibodies for laminin- $\alpha 2$ is often not viable. Therefore, in addition to clinical features (phenotypes, brain MRI, EMG and muscle morphology), molecular genetic testing, such as WES, is highly recommended $(7,35)$. Additionally, WES should be performed for both the patient and their parents, as it assists in improving our understanding of the diagnostic yield for genetically heterogeneous disorders (36), and may result in the identification of novel variants which contribute to this disease (37). With the increase in the use and availability of next generation sequencing techniques, novel variants/mutations associated with MDC1A have been uncovered (7). The newly discovered variant in the present study adds to the genetic spectrum of the disease, and also suggests the contribution of a de novo event to the autosomal recessive inheritance of $\mathrm{MDC} 1 \mathrm{~A}$. Therefore, de novo events should be taken into consideration to accurately determine the occurrence risk of this disease in future offspring.

\section{Acknowledgements}

We are grateful to the patient's family for their participation in and support of this study.

\section{Funding}

The present study was funded by the Vinmec Health Care System (Hanoi, Vietnam; grant no. ISC17.03).

\section{Availability of data and materials}

Data containing information on the variants were deposited on the Global Variome Shared LOVD database (databases. lovd.nl/shared/individuals/00208524). The datasets used or analyzed in the present study are available from the corresponding author on reasonable request.

\section{Authors' contributions}

KTT prepared the experiments and wrote the manuscript. VSL performed the bioinformatics analysis. LTN and CDV performed the clinical diagnoses. All authors have read and approved the final manuscript.

\section{Ethics approval and consent to participate}

Not applicable. 


\section{Patient consent for publication}

Not applicable.

\section{Competing interests}

The authors declare that they have no competing interests.

\section{References}

1. Durbeej M: Laminin- $\alpha 2$ Chain-Deficient Congenital Muscular Dystrophy: Pathophysiology and Development of Treatment. Curr Top Membr 76: 31-60, 2015.

2. Allamand V and Guicheney P: Merosin-deficient congenital muscular dystrophy, autosomal recessive (MDC1A, MIM\#156225, LAMA2 gene coding for $\alpha 2$ chain of laminin). Eur J Hum Genet 10: 91-94, 2002.

3. Philpot J, Sewry C, Pennock J and Dubowitz V: Clinical phenotype in congenital muscular dystrophy: Correlation with expression of merosin in skeletal muscle. Neuromuscul Disord 5 : 301-305, 1995.

4. Colognato $\mathrm{H}$ and Yurchenco PD: Form and function: The laminin family of heterotrimers. Dev Dyn 218: 213-234, 2000

5. Holmberg J and Durbeej M: Laminin-211 in skeletal muscle function. Cell Adhes Migr 7: 111-121, 2013.

6. Xiong H, Tan D, Wang S, Song S, Yang H, Gao K, Liu A, Jiao H, Mao B, Ding J, et al: Genotype/phenotype analysis in Chinese laminin- $\alpha 2$ deficient congenital muscular dystrophy patients. Clin Genet 87: 233-243, 2015.

7. Oliveira J, Gruber A, Cardoso M, Taipa R, Fineza I, Gonçalves A, Laner A, Winder TL, Schroeder J, Rath J, et al: LAMA2 gene mutation update: Toward a more comprehensive picture of the laminin- $\alpha 2$ variome and its related phenotypes. Hum Mutat 39: $1314-1337,2018$

8. Zhou J, Tan J, Ma D, Zhang J, Cheng J, Luo C, Liu G, Wang Y and $\mathrm{Xu} \mathrm{Z}$ : Identification of Two Novel LAMA2 Mutations in a Chinese Patient with Congenital Muscular Dystrophy. Front Genet 9: 43, 2018.

9. Xu B, Ionita-Laza I, Roos JL, Boone B, Woodrick S, Sun Y, Levy S, Gogos JA and Karayiorgou M: De novo gene mutations highlight patterns of genetic and neural complexity in schizophrenia. Nat Genet 44: 1365-1369, 2012.

10. Yu M, Zheng Y, Jin S, Gang Q, Wang Q, Yu P, Lv H, Zhang W, Yuan Y and Wang Z: Mutational spectrum of Chinese LGMD patients by targeted next-generation sequencing. PLoS One 12 : e0175343, 2017.

11. Chae JH, Lee JS, Hwang H, Kim KJ, Hwang YS, Park JD, Cheon JE, Kim IO, Choe GY and Park SH: Merosin-deficient congenital muscular dystrophy in Korea. Brain Dev 31: 341-346, 2009.

12. Dubowitz V, Oldfors A and Sewry C: Muscle Biopsy: A Practical Approach. 4th edition. Saunders Elsevier, 2013

13. Fischer AH, Jacobson KA, Rose J and Zeller R: Hematoxylin and eosin staining of tissue and cell sections. Cold Spring Harb Protoc 2008: pdb.prot4986, 2008.

14. Li H and Durbin R: Fast and accurate short read alignment with Burrows-Wheeler transform. Bioinformatics 25: 1754-1760, 2009.

15. Rimmer A, Phan H, Mathieson I, Iqbal Z, Twigg SRF, Wilkie AOM, McVean G and Lunter G; WGS500 Consortium: Integrating mapping-, assembly- and haplotype-based approaches for calling variants in clinical sequencing applications. Nat Genet 46: 912-918, 2014

16. DePristo MA, Banks E, Poplin R, Garimella KV, Maguire JR, Hartl C, Philippakis AA, del Angel G, Rivas MA, Hanna M, et al: A framework for variation discovery and genotyping using next-generation DNA sequencing data. Nat Genet 43: 491-498, 2011.

17. McKenna A, Hanna M, Banks E, Sivachenko A, Cibulskis K, Kernytsky A, Garimella K, Altshuler D, Gabriel S, Daly M, et al: The Genome Analysis Toolkit: A MapReduce framework for analyzing next-generation DNA sequencing data. Genome Res 20: 1297-1303, 2010.

18. Auton A, Brooks LD, Durbin RM, Garrison EP, Kang HM, Korbel JO, Marchini JL, McCarthy S, McVean GA and Abecasis GR; 1000 Genomes Project Consortium: A global reference for human genetic variation. Nature 526: 68-74, 2015.
19. Cingolani P, Platts A, Wang L, Coon M, Nguyen T, Wang L, Land SJ, Lu X and Ruden DM: A program for annotating and predicting the effects of single nucleotide polymorphisms, SnpEff: SNPs in the genome of Drosophila melanogaster strain $\mathrm{w}^{1118}$; iso-2; iso-3. Fly (Austin) 6: 80-92, 2012.

20. Stenson PD, Ball EV, Mort M, Phillips AD, Shiel JA, Thomas NS, Abeysinghe S, Krawczak M and Cooper DN: Human Gene Mutation Database (HGMD): 2003 update. Hum Mutat 21: 577-581, 2003

21. Adzhubei IA, Schmidt S, Peshkin L, Ramensky VE, Gerasimova A, Bork P, Kondrashov AS and Sunyaev SR: A method and server for predicting damaging missense mutations. Nat Methods 7: 248-249, 2010.

22. Choi Y and Chan AP: PROVEAN web server: A tool to predict the functional effect of amino acid substitutions and indels. Bioinformatics 31: 2745-2747, 2015.

23. Venselaar H, Te Beek TAH, Kuipers RKP, Hekkelman ML and Vriend G: Protein structure analysis of mutations causing inheritable diseases. An e-Science approach with life scientist friendly interfaces. BMC Bioinformatics 11: 548, 2010.

24. Paganoni S and Amato A: Electrodiagnostic evaluation of myopathies. Phys Med Rehabil Clin N Am 24: 193-207, 2013

25. Palisano R, Rosenbaum P, Walter S, Russell D, Wood E and Galuppi B: Development and reliability of a system to classify gross motor function in children with cerebral palsy. Dev Med Child Neurol 39: 214-223, 1997.

26. Hanna SE, Bartlett DJ, Rivard LM and Russell DJ: Reference curves for the Gross Motor Function Measure: Percentiles for clinical description and tracking over time among children with cerebral palsy. Phys Ther 88: 596-607, 2008.

27. Russell DJ, Rosenbaum PL, Cadman DT, Gowland C, Hardy S and Jarvis S: The gross motor function measure: A means to evaluate the effects of physical therapy. Dev Med Child Neurol 31: 341-352, 1989

28. Bohannon RW and Smith MB: Interrater reliability of a modified Ashworth scale of muscle spasticity. Phys Ther 67: 206-207, 1987.

29. Ewing B and Green P: Base-calling of automated sequencer traces using phred. II. Error probabilities. Genome Res 8: 186-194, 1998

30. Le VS, Tran KT, Bui HTP, Le HTT, Nguyen CD, Do DH, Ly HTT, Pham LTD, Dao LTM and Nguyen LT: A Vietnamese human genetic variation database. Hum Mutat 40: 1664-1675, 2019.

31. Hayashi YK, Tezak Z, Momoi T, Nonaka I, Garcia CA, Hoffman EP and Arahata K: Massive muscle cell degeneration in the early stage of merosin-deficient congenital muscular dystrophy. Neuromuscul Disord 11: 350-359, 2001.

32. Sparks SE and Escolar DM: Congenital muscular dystrophies. In: Handbook of Clinical Neurology. Griggs RC and Amato AA (eds). Vol 101. Elsevier, pp47-79, 2011.

33. Canki-Klain N, Béroud C, Clarke NF, Kovac I, Chambert S and Guicheney P: EM.P.3.01 The adult phenotype of congenital muscular dystrophy (MDC1A) due to mutation of LAMA2. Neuromuscul Disord 19: 574, 2009.

34. Jones KJ, Morgan G, Johnston H, Tobias V, Ouvrier RA, Wilkinson I and North KN: The expanding phenotype of laminin $\alpha 2$ chain (merosin) abnormalities: Case series and review. J Med Genet 38: 649-657, 2001.

35. Reddy HM, Cho KA, Lek M, Estrella E, Valkanas E, Jones MD, Mitsuhashi S, Darras BT, Amato AA, Lidov HG, et al: The sensitivity of exome sequencing in identifying pathogenic mutations for LGMD in the United States. J Hum Genet 62: 243-252, 2017.

36. Retterer K, Juusola J, Cho MT, Vitazka P, Millan F, Gibellini F, Vertino-Bell A, Smaoui N, Neidich J, Monaghan KG, et al: Clinical application of whole-exome sequencing across clinical indications. Genet Med 18: 696-704, 2016.

37. Yang Y, Muzny DM, Xia F, Niu Z, Person R, Ding Y, Ward P, Braxton A, Wang M, Buhay C, et al: Molecular findings among patients referred for clinical whole-exome sequencing. JAMA 312: 1870-1879, 2014 